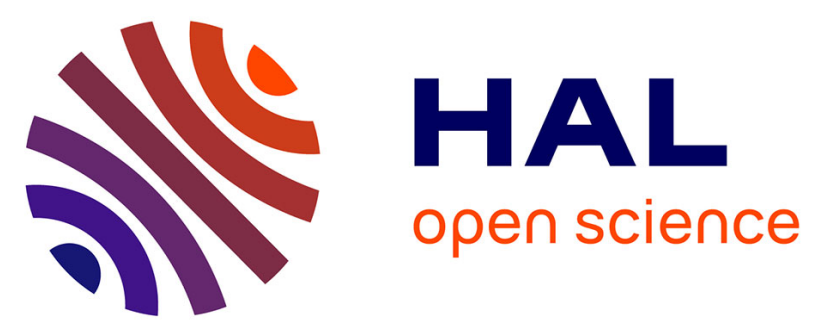

\title{
Etude expérimentale et théorique d'un transistor à flux de vortex réalisé sur couche mince supraconductrice à haute Tc. Comparaison avec un interféromètre haut Tc à boucles multiples
}

\author{
Stéphane Flament, C. Dubuc, Marc Lam Chok Sing, Daniel Bloyet, P. \\ Bernstein, J. Hamet, B. Blanc Guilhon, R. Desfeux
}

\section{To cite this version:}

Stéphane Flament, C. Dubuc, Marc Lam Chok Sing, Daniel Bloyet, P. Bernstein, et al.. Etude expérimentale et théorique d'un transistor à flux de vortex réalisé sur couche mince supraconductrice à haute Tc. Comparaison avec un interféromètre haut Tc à boucles multiples. Journal de Physique III, 1994, 4 (4), pp.627-633. 10.1051/jp3:1994152 • jpa-00249130

\author{
HAL Id: jpa-00249130 \\ https://hal.science/jpa-00249130
}

Submitted on 1 Jan 1994

HAL is a multi-disciplinary open access archive for the deposit and dissemination of scientific research documents, whether they are published or not. The documents may come from teaching and research institutions in France or abroad, or from public or private research centers.
L'archive ouverte pluridisciplinaire HAL, est destinée au dépôt et à la diffusion de documents scientifiques de niveau recherche, publiés ou non, émanant des établissements d'enseignement et de recherche français ou étrangers, des laboratoires publics ou privés. 
Classification

Physics Abstracts

$74.60-74.50$

\title{
Etude expérimentale et théorique d'un transistor à flux de vortex réalisé sur couche mince supraconductrice à haute $T_{\mathrm{c}}$. Comparaison avec un interféromètre haut $T_{\mathrm{c}}$ à boucles multiples
}

\author{
S. Flament ( $\left.{ }^{1}\right)$, C. Dubuc $\left({ }^{1}\right)$, M. Lam Chok Sing $\left({ }^{1}\right)$, D. Bloyet $\left({ }^{1}\right)$, P. Bernstein $\left({ }^{2}\right)$, \\ J. F. Hamet $\left({ }^{2}\right)$, B. Blanc Guilhon $\left({ }^{2}\right)$ et R. Desfeux $\left({ }^{2}\right)$ \\ (') LEI-ISMRA, Bd Maréchal Juin, 14050 Caen Cedex, France \\ (2) CRISMAT-ISMRA, Bd Maréchal Juin, 14050 Caen Cedex, France \\ (Reçu le 15 jullet 1993, révisé le 20 janvier 1994, accepté le 22 février 1994)
}

\begin{abstract}
Résumé. - Nous présentons une étude expérimentale et théorique de structures supraconductrices réalisées sur couches minces d'YBaCuO (déposées sur substrat de $\mathrm{MgO}$ ) commandées en champ magnétique basées, l'une sur le mouvement de vortex Abrikosov, l'autre sur les interférences des fonctions d'onde des paires de Cooper traversant des jonctions Josephson $\mathrm{HT}_{c}$ connectées en parallèle.
\end{abstract}

\begin{abstract}
The theoretical and experimental behaviour of two magnetic field controlled superconducting structures both paterned in a $\mathrm{YBaCuO}$ thin film on $\mathrm{MgO}$ substrate are compared. One is based on the flux flow of Abrikosov vortices and the other one on the interferences between the wave functions of the Cooper pairs crossing high $T_{\mathrm{c}}$ Josephson junctions connected by superconducting loops.
\end{abstract}

\section{Introduction.}

La réalisation de structures amplificatrices facilement commandables est de première nécessité si l'on veut développer une électronique supraconductrice complète. Une méthode séduisante, facilement réalisable sur une couche mince $H T_{c}$, est offerte par la commande en champ magnétique du courant critique d'un ou plusieurs microponts en parallèle [1]. La géométrie et le schéma équivalent (en petits signaux) d'un tel dispositif sont donnés sur les figures 1 et 2.

Le modèle de fonctionnement d'un transistor à flux de vortex Abrikosov est décrit en première partie et confronté aux résultats expérimentaux. Il montre en particulier que la commande du mouvement des vortex par une ligne coplanaire ne permet pas de réaliser un dispositif amplificateur [2].

Dans une deuxième partie, nous présentons une autre structure basée sur les effets Josephson dans des jonctions $\mathrm{HT}_{c}$, dont le principe doit conduire à des gains en courant supérieurs à l'unité. 


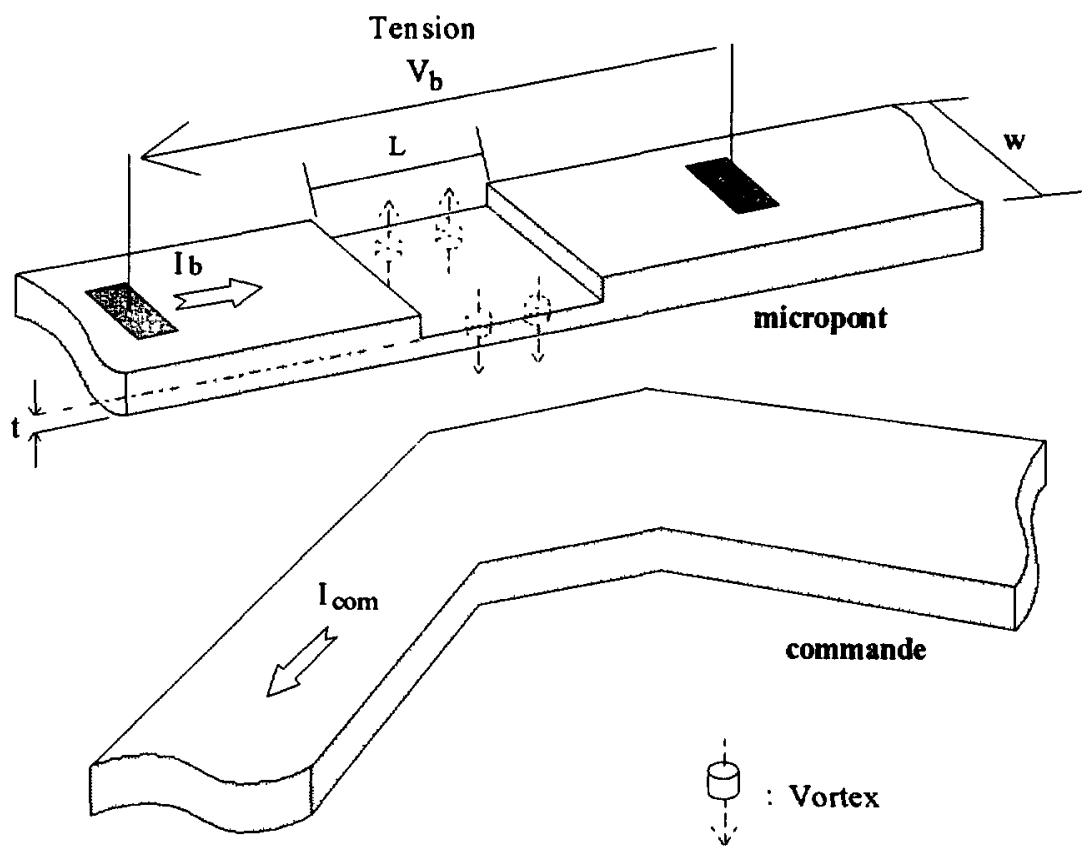

Fig. 1. - Géométrie de l'AVFT étudié. $L=10 \mu \mathrm{m}, W=8 \mu \mathrm{m}, t \approx 80 \mathrm{~nm}$.

[Design of the AVFT.]

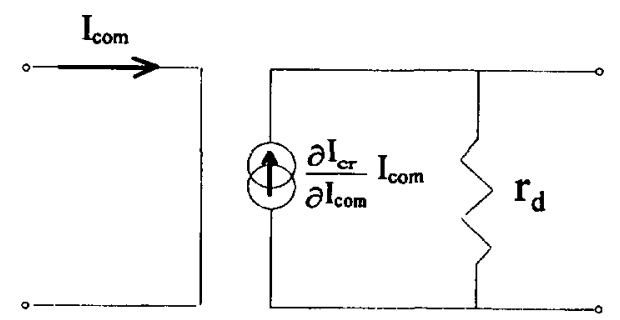

Fig. 2. - Schéma équivalent de l'AFVT en régime quasistatique.

[Equivalent circuit of the AVFT for slow-varying signals.]

\section{Transistor à flux de vortex Abrikosov.}

1.1 PRINCIPE DE FONCTIONNEMENT. - L'explication de la variation du courant critique du micropont passe par une description du mouvement des vortex. La plupart des théories décrivant ce mouvement concernent des situations où l'échantillon est soumis à une excitation magnétique importante, supérieure à $H_{\mathrm{cl}}$, et parcouru par un courant faible [3]. Tous les vortex ont alors le même sens. Dans notre cas, l'excitation magnétique générée par la commande est faible (inférieure à $H_{\mathrm{cl}}$ ) et les fluxons sont générés par le courant de polarisation. Les champs magnétiques créés par ce courant sur chacun des bords du micropont étant de sens opposé, des vortex apparaissent sur un bord et des antivortex sur l'autre (Fig. 1). Nous avons décrit le 
mouvement particulier de ces fluxons à l'aide de la théorie du continuum de l'état mixte [4] qui montre que :

- les moyennes (sur quelques distances intervortex) $\mathbf{B}$ et $\mathbf{J}$ de l'induction locale $\mathbf{b}$ et de la densité de courant locale $\mathbf{j}$ sont reliées par la relation :

$$
\mathbf{B}+\mu_{0} \lambda^{2} \operatorname{rot} \mathbf{J}=n \Phi_{0} \mathbf{u} \quad \text { u vecteur unitaire parallèle au vortex }
$$

- la moyenne temporelle du champ électrique s'écrit :

$$
\mathbf{E}=\mathbf{V}_{\mathrm{L}} \wedge n \Phi_{0} \mathbf{u} \begin{cases}n & \text { densité des vortex } \\ \mathbf{V}_{\mathrm{L}} . & \text { vitesse des vortex }\end{cases}
$$

Lors des mesures, la commande a été systématiquement polarisée en dessous de son courant critique de manière à éviter tout échauffement indésirable.

1.2 Modulation du courant Critique. - Le flux des vortex étant conservatif dans le micropont (il n'y a pas d'accumulation de vortex), div $\left(n \mathbf{V}_{\mathrm{L}}\right)=\mathbf{0}$ et $\mathbf{E}$ est donc constant dans tout le micropont.

Dans l'hypothèse où la force de pinning est négligeable devant la force de Lorentz $\mathbf{j} \wedge \Phi_{0} \mathbf{u}$, générée par le courant de polarisation au moment de la nucléation des vortex, on montre [2] que la valeur absolue de la variation $\Delta I_{\mathrm{cr}}$ du courant critique du micropont s'écrit :

$$
\Delta I_{\mathrm{cr}}=\frac{2 t}{\mu_{0}}\left\|\mathbf{B}_{\mathrm{ext}}(0)-\mathbf{B}_{\mathrm{ext}}(w)\right\|
$$

où $\mathbf{B}_{\text {ext }}$ désigne le champ externe appliqué au micropont, $t$ l'épaisseur de celui-ci, les cotes $y=0$ et $y=w$ désignent les bords du micropont.

Seul un gradient de champ permet donc de moduler le courant critique $I_{\mathrm{cr}}$ du micropont, ce que nous vérifions sur les mesures expérimentales des figures 3 et 4 . On notera en particulier

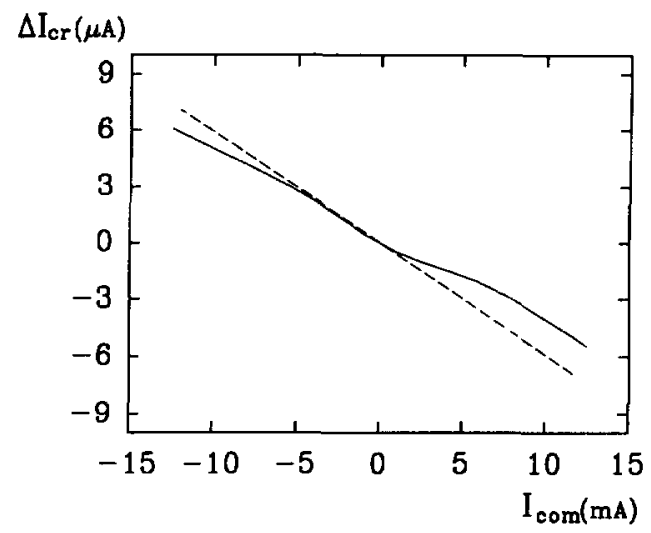

Fig. 3. - Variation théorique (--) et expérimentale du courant critique du micropont par la commande. Pour $1 \mathrm{~mA}$ dans la commande le champ moyen au niveau du micropont est de $9,5 \mu \mathrm{T}$.

[Theoretical (---) and experimental variations of the critical current versus the control current. The mean magnetic field is $9.5 \mu \mathrm{T}$ when $1 \mathrm{~mA}$ flows in the control line.] 


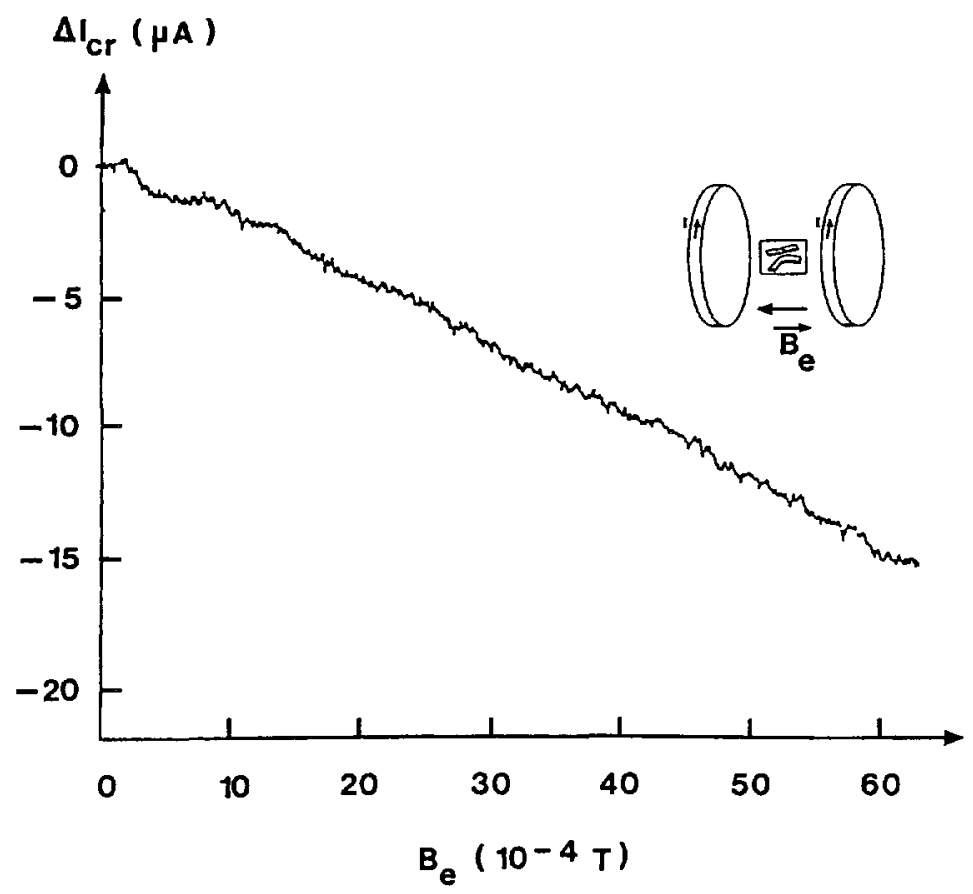

Fig. 4. - Variation expérimentale du courant critique du micropont en champ homogène. A variations de courant critique égales, le champ uniforme à appliquer est beaucoup plus grand que le champ moyen généré par la commande de la figure 3 .

[Experimental variations of the critical current in a uniform field. For equal critical current variations, the applied uniform field has to be much stronger than the mean field created by the control line in figure 3.]

que la variation de $I_{\text {cr }}$ générée par un champ uniforme est très faible en comparaison de celle provoquée par un champ à gradient non nul. Dans le cas où le courant de polarisation $I_{\mathrm{b}}$ et le courant de commande $I_{\text {com }}$ sont à sens contraire (cas de la Fig. 1), il y a diminution du courant critique. Dans le cas contraire, le courant critique est augmenté. Ces conclusions demeurent valides tant que le champ créé par la commande est inférieur à $H_{\mathrm{cl}}$. Au-delà, on retrouve un flux de vortex unipolaires avec diminution du courant critique quel que soit le sens du courant de la commande. Pour le calcul de la variation théorique de $\Delta I_{\mathrm{cr}}$, la commande a été supposée infinie, ce qui conduit à une surestimation du champ qu'elle crée. La valeur exacte nécessiterait l'utilisation d'un logiciel permettant d'obtenir les distributions de courant dans n'importe quelle structure supraconductrice.

1.3 GAIN EN COURANT DE L'AVFT. - Le gain en courant $G_{1}=\frac{\partial I_{\mathrm{cr}}}{\partial I_{\mathrm{com}}}$ vaut :

$$
\left|G_{1}\right|=\frac{\left|\alpha_{\text {com }}(0)-\alpha_{\text {com }}(w)\right|}{\left|\alpha_{\text {bras }}\right|} \approx \frac{\left\|\mathbf{B}_{\text {ext }}(0)-\mathbf{B}_{\text {ext }}(w)\right\|}{\left\|\mathbf{B}_{\mathrm{p}}\right\|}\left|\frac{I_{\mathrm{p}}}{I_{\text {com }}}\right|
$$

où $\alpha_{\text {com }}=\left\|\frac{\partial \mathbf{B}_{\text {ext }}}{\partial I_{\text {com }}}\right\|, \alpha_{\text {bras }}=\left\|\frac{\partial \mathbf{B}_{\mathrm{p}}}{\partial I_{\mathrm{p}}}\right\|$ et $B_{\mathrm{p}}$ désigne l'induction générée au bord de l'échantillon par le courant de polarisation $I_{\mathrm{p}}$. 


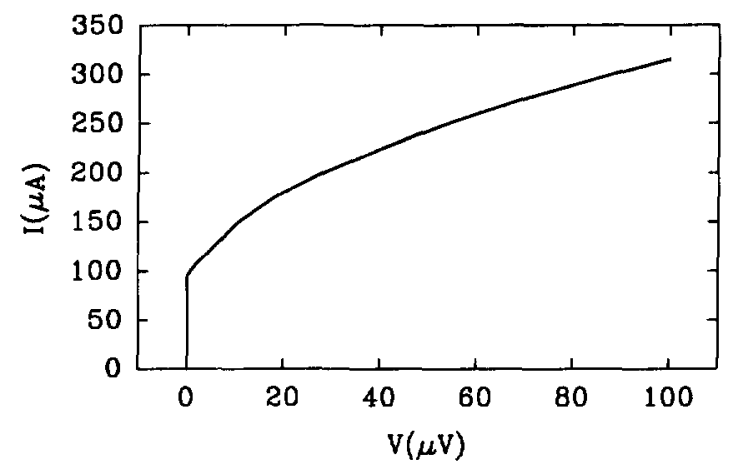

Fig. 5. - Caractéristique $I-V$ typique des microponts commandés.

[Typical $I-V$ characteristics of the controlled microbridges.]

La mesure du gain sur différents échantillons de même géométrie a conduit à une valeur constante de $10^{-3}$; la valeur théorique atteint $6,6 \times 10^{-3}$. L'écart entre ces deux valeurs s'explique par notre impossibilité de pouvoir calculer les valeurs exactes de champ magnétique en tout point et par le fait que, dans nos échantillons, les forces de piégeage ne sont pas négligeables. Les vortex nucléés pour une densité de courant au bord $j_{\mathrm{n}}$ ne se mettent en mouvement que pour une densité de courant $j_{\mathrm{cr}}$ supérieure, dépendant de la force de chaque piège. Ceci se traduit notamment par un arrondissement des caractéristiques $I-V$ (cf. Fig. 5) et une réduction du gain $G_{1}$.

Il est clair qu'à courant $I_{\mathrm{p}}$ et $I_{\text {com }}$ égaux, l'induction générée aux bords de l'échantillon par la commande coplanaire, éloignée de quelques $\mu \mathrm{m}$, est plus faible que celle créée par le courant de polarisation $I_{\mathrm{p}}$. Le coefficient de couplage $\alpha_{\text {com }}$ est donc plus faible que le coefficient $\alpha_{\text {bras. }}$ D'après l'expression (4), on en déduit que le gain en courant $G_{1}$ du dispositif ne peut être supérieur à 1, dans le cas d'une géométrie coplanaire.

\section{Interféromètre à jonctions Josephson.}

Comme le paragraphe précédent nous l'a montré, la limitation théorique principale des performances d'un AVFT résulte de la réponse du dispositif au gradient du champ appliqué. Pour parvenir à une amplification, il faut imaginer un phénomène qui conduise à une sensibilité en champ accrue par rapport à celle rencontrée dans le système à flux de vortex. Un dispositif interférométrique, comprenant plusieurs jonctions Josephson en parallèle, s'affirme comme un bon candidat. En effet, la quantification du flux dans une boucle supraconductrice, interrompue par des jonctions Josephson, provoque l'interférence des fonctions d'onde des paires de Cooper traversant (par effet tunnel ou de proximité) les jonctions et entraîne une variation notable de la différence de phase aux bornes de celles-ci. Or le courant traversant une jonction Josephson est lié justement à la différence de phase aux bornes de celle-ci. Il s'ensuit une modulation importante du courant critique en fonction du champ appliqué à l'interféromètre.

Ce paragraphe est consacré à l'étude des caractéristiques d'un interféromètre (ou multiSQUID) à deux boucles et trois jonctions, commandé par une ligne coplanaire.

2.1 PERFORMANCES THÉORIQUES D'UN MULTI-SQUID. - La modulation maximale du courant critique est obtenue lorsque $\beta=\frac{2 L I_{0}}{\Phi_{0}} \ll 1$ ( $I_{0}$ courant critique des jonctions) [5]. Dans ce 


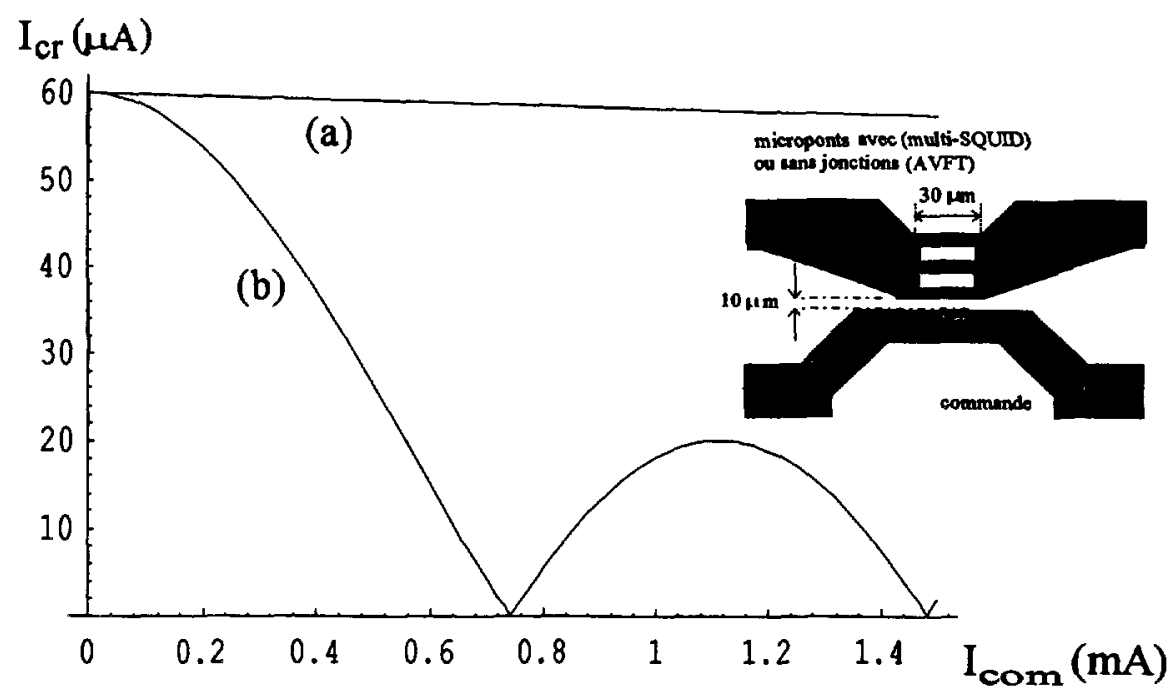

Fig. 6. - Comparaison de la modulation du courant critique de l'AVFT (a) et du multi-SQUID à 3 jonctions (b).

IComparison between the critical current control of the AVFT (a) and the multi-SQUID with 3 junctions (b).]

cas, quand le champ appliqué est le même dans toutes les boucles, le gain $\frac{\partial I_{\mathrm{cr}}}{\partial I_{\mathrm{com}}}$, au point de polarisation $I_{\mathrm{cr}} / 2$, croît comme $n^{2}$ ( $n$ : nombre de jonctions). Un gradient de champ tel que celui créé par la ligne de commande ne modifie pas de façon notable la caractéristique $I_{\mathrm{cr}}\left(I_{\mathrm{com}}\right)$. Nous donnons, sur la figure 6 , à titre d'exemple, les caractéristiques théoriques comparées d'un multi-SQUID et d'un AVFT à deux boucles et trois banches en parallèle. Les courbes ont été tracées pour un courant critique de jonctions de $20 \mu \mathrm{A}$ (valeur typique obtenue sur des jonctions Josephson $\mathrm{HT}_{\mathfrak{c}}$ compte tenu de la géométrie considérée) et un courant critique de l'AVFT de $60 \mu \mathrm{A}$.

2.2 RÉALISATION EXPÉRIMENTALE. - Nous avons réalisé un interféromètre comprenant trois jonctions $\mathrm{HT}_{\mathrm{c}}$ de type « step edge », commandé en champ par une ligne coplanaire. La surface $S$ des boucles est de $25 \times 12 \mu \mathrm{m}^{2}$ (inductance $L=25 \mathrm{pH}$ ), le courant critique mesuré de $60 \mu \mathrm{A}$. Le gain en courant atteint $6 \times 10^{-4}$

Avec trois jonctions identiques, de courant critique ici égal à $I_{0}=20 \mu \mathrm{A}$, le gain en courant théorique $G_{\text {th }}$ se calcule par:

$$
G_{\mathrm{th}}=\frac{\partial I_{\mathrm{cr}}}{\partial I_{\mathrm{com}}}=\frac{\partial I_{\mathrm{cr}}}{\partial B} \frac{\partial B}{\partial I_{\mathrm{com}}}=\frac{\partial I_{\mathrm{cr}}}{\partial \Phi} \cdot S \cdot \frac{\partial B}{\partial I_{\mathrm{com}}}
$$

Avec une efficacité expérimentale de notre commande $\frac{\delta B}{\partial I_{\text {com }}}$ de $3 \mathrm{mT} / \mathrm{A}$ et un transfert théorique $\left.\frac{\partial I_{\mathrm{cr}}}{\partial \Phi}\right|_{\frac{I_{\mathrm{cr}, \mathrm{mdx}}}{2}} \approx \frac{I_{\mathrm{cr}, \max }}{0,5 \Phi_{0}}, G_{\mathrm{th}}$ vaut 0,05 .

Ce gain pourrait être accru par l'amélioration du couplage magnétique (par rapprochement de la ligne de commande notamment) et par augmentation de la surface des boucles. 
L'écart entre les valeurs expérimentales et théoriques s'explique par le manque de reproductibilité de notre processus de fabrication de jonctions. Ceci entraîne une disparité des jonctions du multi-SQUID (en particulier de leur courant critique) et la présence de jonctions élémentaires multiples dans les microponts. Dans ces conditions, des interférences interviennent à la fois à l'intérieur et entre les branches en parallèle. La figure $5 I_{\mathfrak{c}}(\Phi)$ ( $\Phi$ : flux par boucle) est alors le résultat d'un grand nombre d'interférences, dont certaines sont constructives et d'autres destructives et qui provoquent une diminution notable du transfert $\frac{\partial I_{\mathrm{cr}}}{\partial \Phi}$

Satchell et al. [6] ont récemment montré que la maîtrise du processus de fabrication des jonctions Josephson permet d'obtenir des structures interférométriques amplificatrices. Cette équipe a en effet réalisé un interféromètre commandé, comportant cinq jonctions parfaitement identiques (l'identité des jonctions est notamment prouvée par la caractéristique $I_{\mathrm{cr}}(\Phi)$ très proche de la courbe théorique idéale), dont le gain en courant s'établit à 1,7.

\section{Conclusion.}

Si l'on veut obtenir des modulations importantes du courant critique d'un dispositif à flux de vortex Abrikosov, il faut nécessairement exciter celui-ci par des gradients de champ importants, qu'une commande coplanaire ne peut fournir à moins qu'elle soit gravée à une distance submicronique du pont à commander. Actuellement cette technologie n'est pas bien maîtrisée et le multi-SQUID s'affirme alors comme une alternative convaincante. La grande sensibilité au flux magnétique de ce dernier nécessite cependant, en contrepartie, d'isoler complètement le multi-SQUID de son environnement (électrique, thermique et magnétique). Une structure de type AVFT présente, elle, l'avantage d'être intrinsèquement beaucoup moins sensible aux perturbations macroscopiques externes.

\section{Bibliographie}

[1] Hohenwarter G. K. G., Martens J. S., Thompson J. H., Beyer J. B., Nordman J. E., Ginley D. S., Characteristics of superconducting flux flow transistors, IEEE Trans. Magn. 27 (1991) 3297.

[2] Bernstein P., Hamet J. F., Blanc-Guilhon B., Flament S., Dubuc C., Lam Chok Sing M., Bok J., Zhang X. Q., Contour J. P., Ladan F. R., A New Model for the Vortex Flow in HTSC Microbridges, Applied Superconductivity, H. C. Freyhardt Ed., DGM Information-gesellschaft mbH, vol. 2 (1993) p. 1207.

[3] Kim Y. B., Hempstead C. F., Strnad A. R., Flux flow resistance in type II superconductors, Phys. Rev. 139 (1965) 1163.

[4] Hocquet T., Mathieu P., Simon Y., Continuum theory of the mixed state and surface Joule effects in type II superconductors, Phys. Rev. B 46 (1992) 1061.

[5] Likharev K. K., Dynamics of Josephson junctions and circuits (Gordon and Breach Science Publishers).

[6] Satchell J. S., Edwards J. A., Chew N. G., Humphreys R. G., High temperature superconducting vortex flow transistor, Elect. Lett. 28 (1992) 781 ;

Satchell J. S., Humphreys R. G., Edwards J. A., Chew N. G., IEEE Trans. Appl. Supercond. 3 (1993) 2273. 